\title{
COMPARACIÓN ENTRE EL MÉTODO DEL ALGORITMO Y EL MÉTODO GEOMÉTRICO PARA EL CÁLCULO DE ÁREAS DE SECCIONES TRANSVERSALES
}

\section{Comparison between the method of the algorithm and the geometric method for the calculation of areas of cross section}

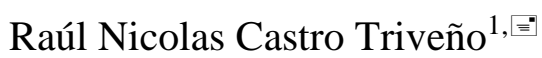 \\ ${ }^{1}$ Universidad Andina del Cusco, Cusco, Perú. \\ Đnicolascastrot@gmail.com
}

\begin{abstract}
Resumen
Objetivos. Describir el método del algoritmo de cálculo de áreas de secciones transversales para terracerías con respecto al método geométrico y determinar el mejor método de cálculo de área de secciones transversales para identificar cual tiene mayor precisión. Materiales y métodos. Se realizó un estudio descriptivo, se consideró dos métodos de estudio, el método del algoritmo y el método geométrico y un método testigo que es el método de las coordenadas realizado en el distrito de Sicuani, en la provincia de Canchis, en el departamento del cusco lugar donde se eligió una vía de estudio la cual se secciono cada 20 metros de los cuales se debía elegir a lazar la muestra para el análisis estadístico y su comparación entre los métodos Resultados. Entre los métodos que se analizaron el método del algoritmo tiene un mejor ajuste ante los cortes de un seccionamiento y de igual manera para el relleno el método del algoritmo tiene un mejor comportamiento que el método geométrico. Conclusiones. Los métodos analizados se ajustan con una mínima diferencia, comportándose mejor el método del algoritmo, siendo entonces una herramienta más eficaz al momento de calcular áreas y siendo esta aplicable para realizar cálculos de corte y relleno.
\end{abstract}

Palabras clave: Método del Algoritmo; Método Geométrico; Seccionamiento; Corte; Relleno.

\begin{abstract}
Objectives. Describe the method of the algorithm of calculation of areas of cross-sections for roads with respect to the geometrical method and determine the best method of calculating area of cross-sections to identify which has greater precision. Materials and methods. A descriptive study was conducted, was considered two methods of study, the method of the algorithm and the geometric method and a method of witness who is the method of the coordinates in the District of Sicuani in Canchis province, in the Department of cusco place where chose a path of study which I I section every 20 metres of them should pick to lazar sample for statistical analysis and comparison between the methods results. Among the methods discussed the method of the algorithm has a better fit before the courts a sectioning as well as for filling algorithm method has a better performance than the geometric method. Conclusions. Analyzed methods conform with a minimal difference, behaving better algorithm method, then being a more effective tool at the time of calculating areas and being this applicable to perform calculations of cutting and filling.
\end{abstract}

Keywords: method of the algorithm; Geometrical method; Sectioning; Court; Filling

Citar como: Castro, R. (2018). Comparación entre el método del algoritmo y el método geométrico para el cálculo de áreas de secciones transversales. Rev Yachay, 7(1),396-403.

Recibido: 16-09-2018; Aceptado 07-12-2018

\section{Introducción}

Los medios de comunicación y transporte entre estos las carreteras desde el inicio de la humanidad han sido el factor más importante para el desarrollo económico, social y militar de una sociedad, constituyendo la principal forma de comunicación, lo que propicia diferentes actividades humanas, por tal motivo es de principal importancia la correcta planeación para la construcción y rehabilitación de estas.

Actualmente el diseño de una carretera involucra varios procedimientos, siendo uno de ellos la determinación de movimiento de tierra que es una actividad constructiva propia de las vías, las que se deben planificar con la mayor precisión posible para permitir un correcto gasto en la construcción de las vías que son parte del desarrollo urbano, social e industrial de un país. 
Consideramos como las principales aplicaciones de las secciones transversales la determinación de áreas de corte y relleno, y por consiguiente los volúmenes de corte y relleno los cuales incidirán en los costos de acarreo y movimiento de tierra, por lo tanto se debe de tener en cuenta aplicar correctamente las herramientas de calculo que tiene carácter geométrico, al calcular las áreas de las secciones por diferentes métodos se requiere especial atención al momento de calcular correctamente las áreas puesto que los volúmenes de corte y relleno se encuentran en función de las áreas de corte y relleno.

\section{Materiales y Métodos}

se realizó un estudio descriptivo en la vía está localizada entre el distrito de Sicuani, en la provincia de Canchis, en el departamento del cusco. Este tramo se inicia en la urbanización Miraflores barrio la florida que corresponde al km. 0 . El levantamiento inicia en el sector de alto calvario con progresiva km. $1+40+0.0$ con una altitud de 3690.165 m.s.n.m con coordenadas norte: 8420854.577 este: 260208.572, y finaliza en el sector de Rosaspata alto con progresiva km. 0+90+0.0 con una altitud 3649.826 m.s.n.m con coordenadas norte: 8420429.274 este: 260435.867. Considerando materiales como clavos, estacas, fichas, yeso, Eclímetro cts berger 5 1/4” abney level, Estación total topcon gts-211d, Gps navegador garmin gpsmap 76csx, nivel de ingeniero Nikon ac-2s

Se realiza en 2 fases donde en la primera etapa se realiza reconocimiento, establecimiento de bm's, levantamiento topográfico y la verificación y corrección y una segunda etapa donde se realizó los cálculos, planos, cálculo de áreas, verificación y corrección y los trabajos de gabinete correspondiente a la comparación de ambos métodos.

Para desarrollar la información y obtención de datos se realiza un levantamiento topográfico donde se pone preponderancia el seccionamiento que es la determinación de las secciones de carretera, es un procedimiento sencillo pero laborioso, ya que a cada veinte metros de nuestra línea del camino, se tendrá que determinar, diez metros a la izquierda y diez metros a la derecha. El objetivo que sean veinte metros es abarcar el área de estudio con respecto del eje.

Posteriormente se realiza los cálculos del área de las secciones para lo cual existen varios métodos manuales que eventualmente pueden ser utilizados y que son la base analítica de las técnicas computacionales:

El método de las figuras geométricas es donde las secciones transversales se dividen en figuras geométricas conocidas generalmente triángulos, rectángulos y trapecios, para así calcular el área de cada una de ellas separadamente.

Figura 1. Área de sección transversal por figuras geométricas

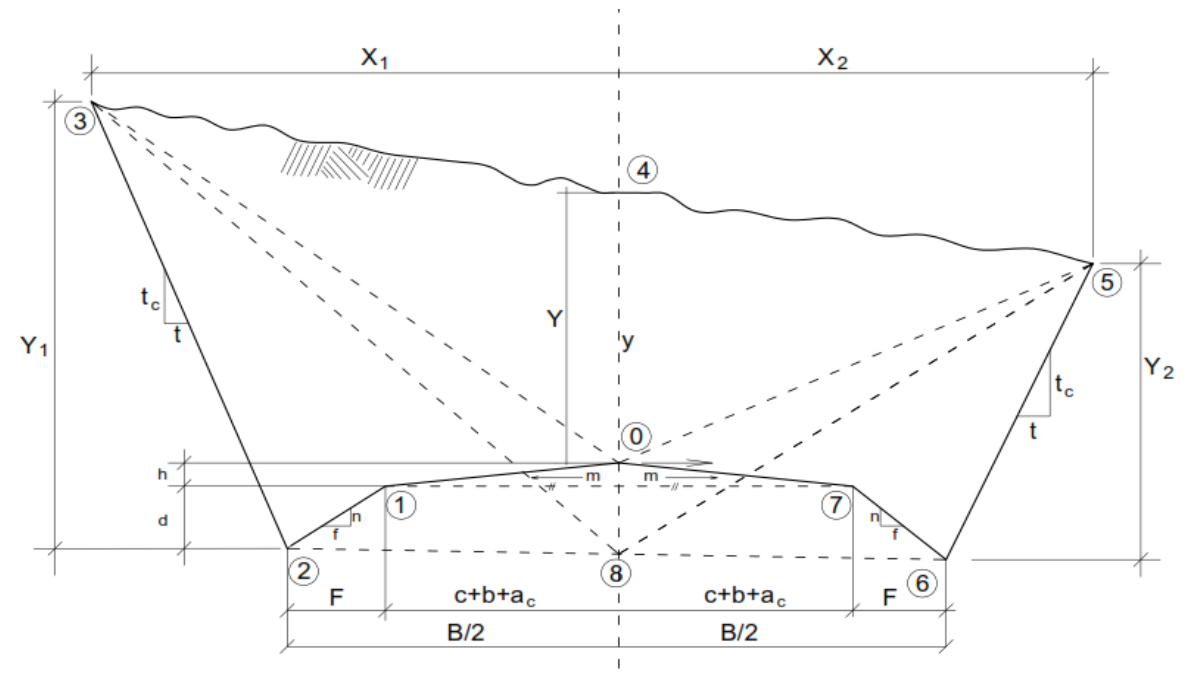

Fuente: diseño geométrico de carreteras (cárdenas et. al.).

El método de las coordenadas de los vértices donde se utiliza un sistema de coordenadas (x,y), de origen la cota roja en el eje de la vía, de tal manera que la suma de los productos y por x de las líneas continuas, menos la suma de los productos y por x de las líneas discontinuas, arrojan como resultado el doble área (cárdenas et. al.). 
Figura 2. Área de sección transversal por las coordenadas de los vértices

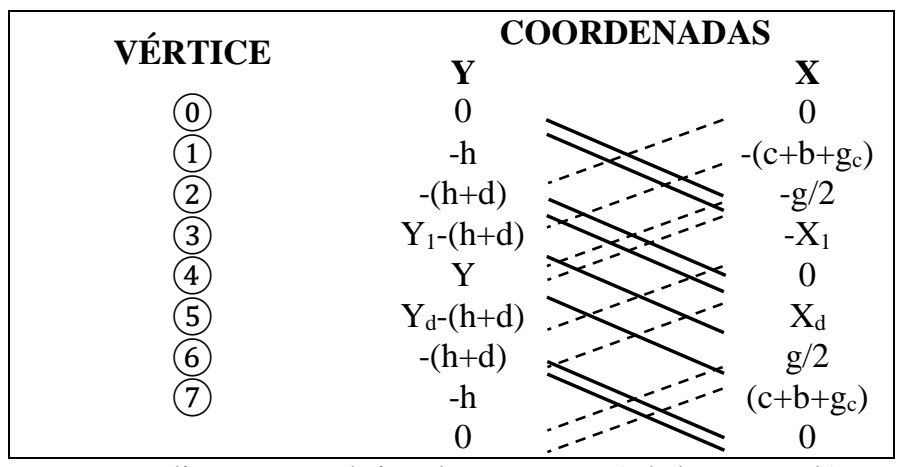

Fuente: diseño geométrico de carreteras (cárdenas et. al.).

EL método del algoritmo es un algoritmo para el cálculo de áreas totales de excavación (corte) y relleno (terraplén) de una sección transversal de construcción de una carretera, este desarrollo parte de la conceptualización de la sección transversal como una superposición de dos perfiles definidos mediante puntos $(\mathrm{x}, \mathrm{y})$, aplicando conceptos de cálculo integral y funciones discretas para la identificación de figuras simples que hagan práctica la obtención de las áreas totales.

El cálculo de las áreas totales de corte y terraplén de una sección transversal de carretera dados dos perfiles superpuestos conocidos: el terreno y el perfil de construcción de define sistemáticamente:

Proporcionar todos los puntos conocidos del terreno. Esta información se obtiene de la libreta de nivelación para secciones transversales. Se tendrá que adaptar para formar un arreglo que contenga a los puntos (x, y) que definen e perfil del terreno.

Proporcionar todos los puntos conocidos del perfil de construcción. esta información se obtiene del diseño de la carretera tomando en cuenta las pendientes de drenaje, el ancho de la superficie de rodamiento, la geometría y dimensiones de la cuneta y la contra cuneta, así como las pendientes de los taludes de corte del terreno y terraplén del material utilizado para el relleno.

Completados el paso 1 y el paso 2 se procede a realizar el recorte de los dos perfiles encontrando los puntos de intersección inicial y final es decir los "ceros".

Se aplica el procedimiento de normalización a ambos perfiles insertando puntos faltantes correspondientes para cada perfil. Al final de este paso ambos perfiles deben de tener el mismo número de puntos, además todos los puntos deberán de tener su correspondiente en el otro perfil compartiendo la misma abscisa.

Inicializar a cero dos acumuladores. Uno para el área total de corte y otro para el área total de terraplén de la sección transversal.

Se inicia este paso tomando el primer y siguiente punto de ambos perfiles. Se obtienen las diferencias en las ordenadas (cy) y la diferencia en las abscisas (dx). se identifica a que figura pertenece por medio de los signos y los valores de las diferencias de las ordenadas y se calculan las áreas, se suman los valores obtenidos a los acumuladores de área inicializados en el paso 5, dependiendo de si es corte o terraplén.

Se repite el paso 6 hasta que se analizan todos los puntos de ambos perfiles.

Este es el paso final. Los valores de área total obtenidos en corte y terraplén se encuentran en los acumuladores que se inicializaron en el paso 5 y se fueron acumulando a lo largo de las iteraciones entre el paso 6 y el 7.

Rev. Yachay volumen (7) Número (1), enero-diciembre 2018 
Tabla 1

Fórmulas de cálculo de área para figuras simples

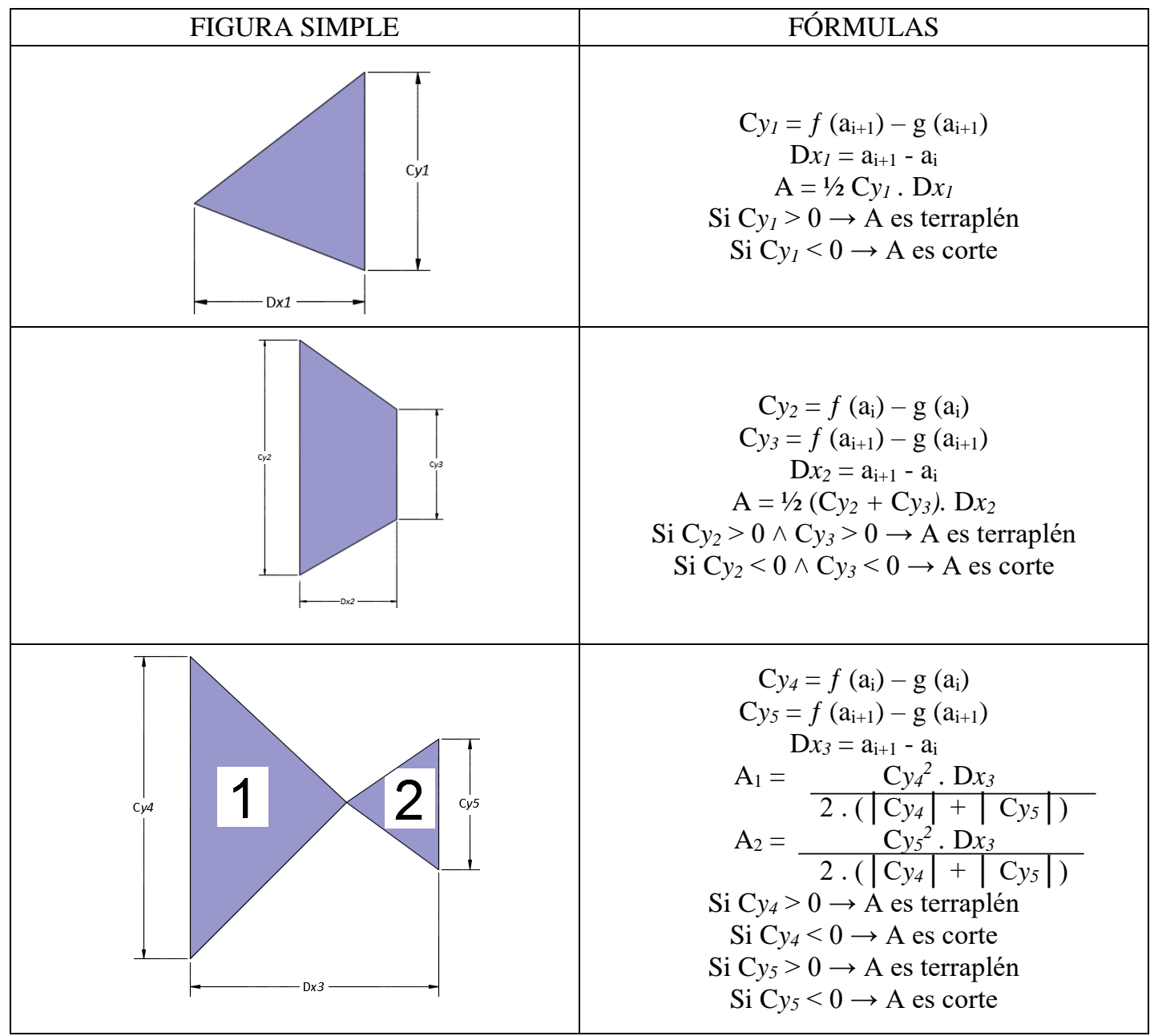

Fuente: algoritmo de cálculo de áreas de secciones transversales para terracerías de un proyecto geométrico de carreteras (grife, salomón, sánchez et. al.) 


\section{Resultados}

Para la determinación de la muestra, se utilizó el muestro aleatorio simple (mas), en el cual se extrajo 10 muestras aleatoriamente según la tabla siguiente:

\begin{tabular}{|ll}
\hline INDICE & DATOS \\
\hline 1 & $0+16+00$ \\
2 & $0+20+00$ \\
\hline 3 & $0+46+00$ \\
4 & $0+04+00$ \\
5 & $0+02+00$ \\
6 & $0+40+00$ \\
7 & $0+36+00$ \\
8 & $0+12+00$ \\
9 & $0+14+00$ \\
\hline 10 & $0+38+00$ \\
\hline
\end{tabular}

Resultado de áreas Para cada sección se realizará por tres métodos considerando el método de las coordenadas como testigo para clarificar los resultados por áreas obtenidos por cada método: $\mathrm{t} 1$ método geométrico, t2 método de coordenadas (testigo), t3 método del algoritmo

Los resultados se reflejan por método en la tabla ${ }^{\circ} 2$ :

Áreas en $\mathbf{m} 2$ por método

\begin{tabular}{lllllll}
\hline & CORTE & RELLENO & CORTE & RELLENO & CORTE & RELLENO \\
\hline $0+16+0$ & 8.386 & 1.141 & 8.387 & 1.143 & 8.387 & 1.142 \\
$0+20+0$ & 17.453 & 1.652 & 17.800 & 1.872 & 17.452 & 1.651 \\
$0+46+0$ & 19.134 & & 19.132 & & 19.132 & \\
$0+04+0$ & 2.097 & 4.238 & 2.072 & 4.484 & 2.072 & 4.483 \\
$0+02+0$ & 12.175 & 1.711 & 12.118 & 1.716 & 12.118 & 1.711 \\
$0+40+0$ & 3.721 & 4.981 & 3.672 & 4.982 & 3.381 & 4.982 \\
$0+36+0$ & 5.021 & 1.818 & 5.025 & 1.818 & 5.024 & 1.818 \\
$0+12+0$ & 3.278 & 4.457 & 3.277 & 4.456 & 3.276 & 4.457 \\
$0+14+0$ & 2.836 & 1.348 & 2.853 & 1.348 & 2.853 & 1.348 \\
$0+38+0$ & 2.225 & 2.274 & 2.225 & 2.274 & 2.224 & 2.255 \\
\hline
\end{tabular}

Rev. Yachay volumen (7) Número (1), enero-diciembre 2018 
Análisis de varianza de corte (RBD) tabla nº

\begin{tabular}{llccccc}
\hline $\begin{array}{l}\text { Origen de las } \\
\text { variaciones }\end{array}$ & $\begin{array}{l}\text { Sumade } \\
\text { cuadrados }\end{array}$ & $\begin{array}{l}\text { Grados de } \\
\text { libertad }\end{array}$ & $\begin{array}{c}\text { Promediode los } \\
\text { cuadrados }\end{array}$ & $F$ & $\begin{array}{c}\text { Probabilidad } \\
\text { Valorcrítico } \\
\text { paraF }\end{array}$ \\
\hline PROGRESIVAS & 1130.735 & 9 & 125.637 & 17428.900 & 0.00 & 2.456 \\
METODO & 0.021 & 2 & 0.011 & 1.464 & 0.2576575 & 3.555 \\
ERROR & 0.128 & 18 & 0.007 & & & \\
TOTAL & 1130.886 & 29 & & & \\
$\quad$ CV\% & $1.11 \%$ & & & & \\
\hline
\end{tabular}

Dado que la probabilidad calculada en la tabla de análisis de varianza para la variable corte es mayor a 0.05 , se concluye que no hay diferencias significativas entre los métodos utilizados para hallar el área de corte de las secciones transversales, por lo que se acepta la hipótesis nula ya que el uso de cualquiera de estos métodos no influye significativamente en la variación de las áreas en metros cuadrados (m2) rechazando así la hipótesis alterna.

Este factor tiene un efecto estadísticamente no significativo en el cálculo del área en metros cuadrados (m2) con un nivel de confianza del 95\% según la regla de decisión por lo que se realizó la prueba de rango múltiple de tukey (hsd=0.079). El coeficiente de variación, es confiable lo que significa que de cada 100 repeticiones, el $1.11 \%$ van a salir diferentes y el $98.89 \%$ de observaciones serán confiables, es decir serán valores iguales.

Tabla 4

Prueba tukey para el corte

\begin{tabular}{llll}
\hline & T2 & T1 & T3 \\
& $(7.656)$ & $(7.633)$ & $(7.592)$ \\
\hline T2 (7.656) & ------- & 0.023 & 0.064 \\
T1 $(7.633)$ & ------- & ------ & 0.041 \\
T3 (7.592) & -------- & ------- & ------ \\
\hline
\end{tabular}

Los resultados de la prueba de rango múltiple de tukey indican que no existen diferencias significativas entre los métodos, en conclusión los métodos de cálculo para área de corte son similares con una diferencia significativa mínima, lo que quiere decir que el algoritmo de cálculo se ajusta a los dos métodos de cálculo con diferencias mínimas. el análisis muestra que existe una mayor diferencia entre el método del algoritmo, con respecto al método geométrico y por coordenadas, en conclusión el método del algoritmo con respecto al geométrico tiene un mejor ajuste para el cálculo de áreas para secciones transversales, siendo este método alternativo para el cálculo de áreas para secciones transversales en corte.

ANÁLISIS DE VARIANZA RELLENO (RBD) Tabla 5:

\begin{tabular}{llccccl}
\hline $\begin{array}{l}\text { Origen de las } \\
\text { variaciones }\end{array}$ & $\begin{array}{l}\text { Sumade } \\
\text { cuadrados }\end{array}$ & $\begin{array}{l}\text { Grados de } \\
\text { libertad }\end{array}$ & $\begin{array}{l}\text { Promedio de los } \\
\text { cuadrados }\end{array}$ & $F$ & Probabilidad & $\begin{array}{l}\text { Valor } \\
\text { crítico para } \\
F\end{array}$ \\
\hline PROGRESIVA & 73.899 & 9 & 8.211 & 2396.868 & 0.000 & 2.456 \\
METODO & 0.011 & 2 & 0.006 & 1.634 & 0.22287495 & 3.555 \\
ERROR & 0.062 & 18 & 0.003 & & & \\
TOTAL & 73.972 & 29 & & & & \\
$\quad$ CV\% & $2.45 \%$ & & & & & \\
\hline
\end{tabular}


Dado que la probabilidad calculada en la tabla de análisis de varianza para la variable corte es mayor a 0.05 , se concluye que no hay diferencias significativas entre los métodos utilizados para hallar el área de relleno de las secciones transversales, por lo que se acepta la hipótesis nula ya que el uso de cualquiera de estos métodos no influye significativamente en la variación de las áreas en metros cuadrados (m2) rechazando así la hipótesis alterna.

Este factor tiene un efecto estadísticamente no significativo en el cálculo del área en metros cuadrados ( $\mathrm{m} 2)$ con un nivel de confianza del 95\% según la regla de decisión por lo que se realizó la prueba de rango múltiple de tukey (hsd=0.051). El coeficiente de variación, es confiable lo que significa que de cada 100 repeticiones, el $2.45 \%$ van a salir diferentes y el $97.55 \%$ de observaciones serán confiables, es decir serán valores iguales.

Tabla 6

Prueba tukey para el relleno

\begin{tabular}{llll}
\hline & T2 & T3 & T1 \\
& 2.677 & 2.650 & 2.624 \\
\hline T2 $(2.677)$ & ------- & 0.027 & $0.053^{*}$ \\
T3 $(2.650)$ & ------- & ------- & 0.025 \\
T1(2.624) & ------- & ------ & ----- \\
\hline
\end{tabular}

Los resultados de la prueba de rango múltiple de tukey indican que existen diferencias significativas entre el método geométrico y por coordenadas lo que quiere decir que existe una diferencia entre las áreas de relleno entre ambos métodos, así mismo no existe diferencias significativas entre el método de coordenadas y geométrico con respecto al algoritmo de cálculo, en conclusión los métodos de cálculo para área de relleno son similares con una diferencia significativa mínima, lo que quiere decir que el algoritmo de cálculo se ajusta a los dos métodos de cálculo con diferencias mínimas. Por consiguiente se muestra que existe una mayor diferencia del método de coordenadas, con respecto al método geométrico y algoritmo, en conclusión el método del algoritmo con respecto al geométrico tiene un mejor ajuste para el cálculo de áreas para secciones transversales, siendo este método alternativo para el cálculo de áreas para secciones transversales en relleno.

\section{Discusión}

El método del algoritmo al ser de fácil aplicación, es de fácil implementación computacional, y falta incorporar otros elementos constructivos propios de una vía como son las cunetas. El método de cálculo de este algoritmo se ajusta a figuras geométricas simples lo que es aplicable en el método geométrico, con excepción del triángulo doble, lo que supone que es una figura de área mixta (corte relleno) en el cual se evalúa a la vez dos áreas y el sistema de ingreso de la sección transversal del algoritmo es de manera sencilla, pero se debe detallar el desnivel total con respecto a la distancia horizontal acumulada lo que supone un cálculo previo de dicha sección transversal. El cálculo de la superficie de construcción contempla hallar las coordenadas de los detalles en cada punto, este método no debe de confundirse con el método de coordenadas, puesto que solo se utiliza para hallar el espesor y las distancias de las particiones de cada figura geométrica. Si bien es cierto que este método utiliza la continuidad de funciones para hallar las áreas se debe tener en cuenta que toda sección es continua dentro del intervalo del talud de corte, plataforma y talud de relleno. También se debe evaluar este algoritmo con diferentes métodos de levantamiento de secciones, para evaluar con qué tipo de levantamiento se observa un mejor ajuste al calcular áreas de corte y relleno.

\section{Conclusiones}

El método propuesto por Zaragoza grife (profesor investigador del cuerpo académico de construcción fiuady), Alonso salomón (profesor de carrera del cuerpo académico de geotecnia fiuady), López Sánchez (profesor de carrera del cuerpo académico de ciencias básicas fiuady), se ajusta al método geométrico de cálculo de secciones transversales, con una mínima diferencia entre ellos, suponiendo una herramienta más eficaz al momento de calcular áreas de corte y relleno. Se identifica que los errores de cálculo de áreas entre el método del algoritmo con respecto al método geométrico son mínimos en corte y relleno, corroborándose en el análisis estadístico, se refleja que entre estos métodos no existe diferencias significativas lo que quiere decir que este método es una herramienta válida de cálculo de áreas. 
Se observó una diferencia significa entre el método de coordenadas y los otros dos métodos, lo cual supone que es una herramienta válida pero conllevaría a mayores gastos en movimiento de tierras.

\section{Bibliografía}

Alonzo salomón, lauro Ariel; Rodríguez Rufino Gabriel. (2005). Carreteras. Yucatán. Ediciones de la universidad autónoma de Yucatán.

Cárdenas Grisales, james. (2004) Diseño geométrico de carreteras. Colombia. ECOE ediciones.

Crespo Villalaz, Carlos. (2004) Vías de comunicación caminos, ferrocarriles, aeropuertos, puentes y puertos. México. Tercera edición. Editorial Limusa.

Dirección general de caminos y ferrocarriles DGFC. (2005) Manual para el diseño de caminos no pavimentados de bajo volumen de tránsito. Perú. Directoral n 084-2005- MTC/14 del 16.11.2005.

García Márquez, Fernando. (2003). Curso básico de topografía planimetría, agrimensura y altimetría. México. Editorial PAX México.

García Martin, Antonio; Rosique Campoy, Manuel y Segado Vásquez francisco. (1994) Topografía básica para ingenieros. España. Servicio de publicaciones universidad de Murcia.

Gonzales Cabezas, Antonio miguel. (2010). Lecciones de topografía y replanteos. España. Quinta edición, editorial club universitario.

López Gayarre, Fernando. (2006) Elementos de topografía y construcción. España. Tercera edición, ediciones de la universidad de Oviedo.

Ministerio de transportes y comunicaciones. (2013) Manual de carreteras Diseño geométrico DG- 2013. Perú. Dirección general de caminos y ferrocarriles.

Morales Sosa, Andrés. (2006) Ingeniería vial I para estudiantes de grado de ingeniería civil. Perú. Editora búho.

Organización de las naciones unidas para la agricultura y la alimentación (fao). (1950) Manual de campo para la ordenación de cuencas hidrográficas diseño y construcción de caminos en cuencas hidrográficas frágiles. Roma. Guía conservación 13/5.

Zaragoza Grife, J.N.; Alonso salomón, 1. y López Sánchez, r. (2010) Algoritmo de cálculo de áreas de secciones transversales para terracerías de un proyecto geométrico de carreteras. España. Facultad de ingeniería de la universidad autónoma de Yucatán. 